\title{
Unmasking forest borderlines by an automatic delineation based on airborne laser scanner data
}

\author{
A. Alivernini ${ }^{a, b}$, A. Barbati ${ }^{b}$, S. Fares ${ }^{a}$ and P. Corona ${ }^{c}$ \\ aConsiglio per la ricerca in agricoltura e l'analisi dell'economia agraria, Research Centre for the Soil-Plant

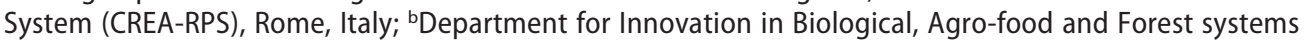 \\ (DIBAF), University of Tuscia, Viterbo, Italy; 'Consiglio per la ricerca in agricoltura e l'analisi \\ dell'economia agraria, Forestry Research Center (CREA-SEL), Arezzo, Italy
}

\begin{abstract}
This study presents an automatic methodology based on airborne laser scanner (ALS) data, which allows the mapping of forests, using quantitative criteria typical of forest definitions, i.e. minimum threshold for the height of trees, canopy cover, forest area size, and width. Interactions between forest and other land uses are explored by the methodology for the definition of forest borderlines using an additional criterion; this criterion is the distance-discontinuity (DD), which establishes a minimum width (MW) for portions of territory categorized by land uses different from the forest. The proposed forest mapping approach introduces also a fuzzy algorithm to assess the canopy cover, thereby enhancing the positional accuracy in the delineation of the forest borderline. This methodology has a very flexible mapping approach with the potential to address the many forest definitions existing worldwide. The evaluation and the improvement of the methodology are particularly encouraged by its release as an open source tool.
\end{abstract}

\section{ARTICLE HISTORY}

Received 9 January 2015

Accepted 6 June 2016

\section{Introduction}

Data on forests' spatial distribution are crucial for forest protection and conservation, spatial pattern analysis, climate change research, and other forest-related applications (Barbati et al. 2014; Corona et al. 2011). Currently, several approaches for forest mapping exist, which are different in terms of spatial scales (from local to global), minimum mapping units, level of thematic detail (e.g. boolean forest masks or, on the contrary, forest-type maps), sources of information, etc. (Corona 2010). In addition, forest mapping must always consider that a plethora of forest definitions exist (over 800 terms worldwide; Lund 2014). Even within Europe, national forest inventories data are still collected using diversified forest definitions (Vidal et al. 2008).

Forest definitions are based on both quantitative and qualitative criteria. The quantitative information needed to classify forest versus non-forest land is mainly represented by the height of trees, the minimum size of the forest area, the minimum width of linear

CONTACT A. Alivernini $\otimes$ alessandro.alivernini@crea.gov.it $\Theta$ Consiglio per la ricerca in agricoltura e l'analisi dell'economia agraria, Research Centre for the Soil-Plant System (CREA-RPS), Rome, Italy 